\title{
Comparison of experimental study of concentration measurement using pLIF and EIT
}

\author{
D. Jasikova ${ }^{1, \text { a }}$, M. Kotek ${ }^{2}$, D. Krcmarik ${ }^{2}$, V. Kopecky ${ }^{1}$ \\ ${ }^{1}$ Faculty of Mechatronics, Informatics and Interdisciplinary Studies, Technical University of Liberec, 46117 Liberec, \\ Czech Republic \\ ${ }^{2}$ Institute for Nanomaterials, Advanced Technologies and Innovation, Technical University of Liberec, 46117 Liberec, \\ Czech Republic
}

\begin{abstract}
In this paper we show the results of the comparison study. The study deals with the planar Laser Induced Fluorescence (pLIF) and Electrical Impedance tomography (EIT) method. Both methods were used for the visualisation of concentration changes in basic mixing process.
\end{abstract}

\section{Introduction}

The monitoring of the multiphase flow has been an important part of measurement technology in many industrial branches such as medical industry, petro chemistry, water service, pharmaceutical and others. The Electrical Impedance Tomography (EIT) system has many advantages such as low cost, simple structure, noninvasive approach.[1]

The technical merit of the EIT system lies in the possibility of obtaining the 3D spatial distribution of the substance that can be taken in one time step. The EIT system is an imaging technique based on the electrical conductivity of the matter in the response to the applied electrical current. The injecting current corresponds to the measurement frequency (the frequency changes for different substances). The multi frequency EIT system allows operating with 16 electrodes within one measuring plane with maximum total frequency of $50 \mathrm{~Hz}$. This setup is suitable for industrial process application especially for multiphase flow. The setup with 4 planes, each with 16 electrodes for 3D measurement offers image reconstruction of both conductivity and permittivity distribution in 3D. [2]

The typical EIT applications are mixtures, fluid flow measurements, phase separation processes, filtration, chemical reaction or concentration processes. The conductivity is also temperature dependent - using EIT method we can study temperature changes or it can be used in multiphase flow visualisation in $3 \mathrm{D}$ mode.

\footnotetext{
a darina.jasikova@tul.cz
}

Also exist an optical way to study temperature, concentration or $\mathrm{pH}$ distribution of the flow. The optical access is non-invasive but some authors [3] doubt about the intrusiveness part of the measurements especially for processes with low temperature fields where the results can be influenced by the laser light energy.

The advantage of the optical methods is the $2 \mathrm{D}$ spatial resolution that is comparable to the resolution of the CCD chip of the imaging part of the experimental setup.

In this paper we present two techniques for observation of mixing processes. The EIT is particular useful for substances and vessels which are not transparent. The EIT technique is dependent on the initial conductivity of the examined object. The initial conductivity information influences the current and frequency setup and it has also direct effect on the final results. On the other hand the planar Laser Induced Fluorescence (pLIF) method does not require initial conductivity information of the continuous substance but requires transparency of both vessel and substance. The mixing process is measured by both techniques simultaneously, and presented experiment shows similar results.

\section{Experimental methods}

\subsection{Planar Laser Induced Fluorescence}

The planar laser-induced fluorescence (pLIF) is a technique based on a well-defined relationship between the intensity of the fluorescence and the concentration, while other experimental parameters constant. 
The fluorescence physically corresponds to an atomic de-excitation process with emission of a photon of lower energy than the one which caused the excitation. When the matter is enlightened it re-emits light at a longer wavelength $\lambda_{\text {em }}$ than the excitation wavelength $\lambda_{\text {ex }}$. The captured fluorescence is filtered out to reduce the excitation light. For this purpose is used the shift between excitation and emission spectra. This is enabled by optical filters $(550 \mathrm{~nm})$ that let only the re-emitted light to enter the detector.

Rhodamine 6G dye was used to observe the concentration changes. This dye is highly soluble in water and is very chemically stable for very large range of concentration levels. The quantum efficiency of Rhodamine is very high, so it can give us very good fluorescence signals. The spectral characteristics of the dye are close to the Nd:YAG lasers (the lasers with wavelength $532 \mathrm{~nm}$ ). This wavelength is close to the optimal excitation wavelength of the Rhodamine 6G. Here we set the maximum concentration level on $0.003 \%$. The calculation of the results based on the calibration curve that responds to the dependence between the laser light intensity, emitted light intensity that is represent by the precisely concentration value.

This calibration measurement was made with precisely known concentration value of the substance.

\subsection{Electrical Impedance Tomography}

Here we used EIT system, manufactured by ITS especially for the purpose of the 3D measurement with high spatial resolution. This system used is the model $\mathrm{p} 2+$. The electrode system was fitted on the experimental vessel, fabricated from optically clear polyacrylic plastic.

The sensors were divided in 4 planes, each plane settled with 16 sensors. The planes were $100 \mathrm{~mm}$ from each other. The tomography allows options to be adjusted which can dramatically improve the measurement [4]. It enables averaging and delay cycles. These two parameters help to avoid local discrepancies in the conductivity during measurement. It also allows defining different vessel types. The vessels can be either non-conductive (as in this paper described) or conductive. In the case of conductive vessel it is necessary to have isolated the electrodes from the wall of the vessel. Anyway in both cases the electrodes have to be in direct contact with measured substance. The sensor geometries depend on the vessel shape. Possible sensor geometries are in Fig. 1.

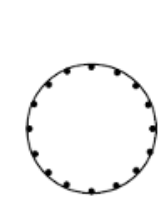

(a) Circular
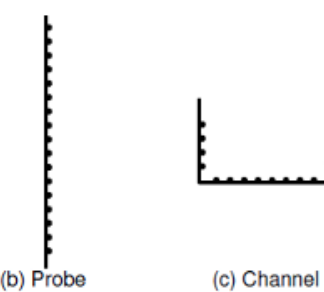

(c) Channel

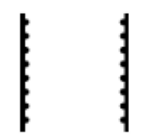

(d) Dual-probe
Fig. 1. Different sensor geometries: a) circular, b) probe, c) channel, d) dual-probe.

\section{Experimental setup}

The experiment was performed in a cylindrical vessel with radius $7.5 \mathrm{~cm}$. The vessel was filled with water up to $34 \mathrm{~cm}$ (resulting in total volume of $6 \mathrm{l}$ ). The pLIF technique and EIT system were synchronized from common triggering source Keithley 3390 with repetition period of $1 \mathrm{~s}$. The experimental setup can be seen on figure 2 .

We divided the investigation into two parts; the first experiment assumed the basic mixing process of $0.003 \%$ $\mathrm{NaCl}$ solution in tapped water. For the purpose of $\mathrm{pLIF}$ method the $\mathrm{NaCl}$ solution was coloured with concentration sensitive fluorescent dye Rhodamine 6G. This experiment should prove the diffusion effect of $\mathrm{NaCl}$ solution in the tapped ware.

The second part of experiment was focused on vortex structures initiated with magnetic stirrer $-450 \mathrm{rmp}$. The working liquid was again the mixture of $\mathrm{NaCl}$ in water with Rhodamine $6 \mathrm{G}$ in tapped water. The results of experiments helped to compare the advantages of both methods. We synchronized both methods to get comparable results that were investigated and validated.

In both cases the EIT system current was set at $4.7-$ $4.11 \mathrm{~mA}, 19200 \mathrm{~Hz}$. During the measurement the AC current was applied between two electrodes and simultaneously resulting voltage between other pairs of electrodes was measured at the same time.

In our experiment we used tapped water from mains as a continuous phase with conductivity of $0.16 \mathrm{mS} / \mathrm{cm}$. The second substance was $310^{-3} \%$ concentration of $\mathrm{NaCl}$ in water coloured with Rhodamine $6 \mathrm{G}$ in concentration $0.003 \%$ with conductivity of $4 \mathrm{mS} / \mathrm{cm}$. The Rhodamine was used in order to track the mixing by the means of pLIF method. The solution was used in the same concentrations in order to track the mixing process by the means of tomography. Data were taken from the third plane (measured from the top) which means $20 \mathrm{~cm}$ from the bed of the vessel.

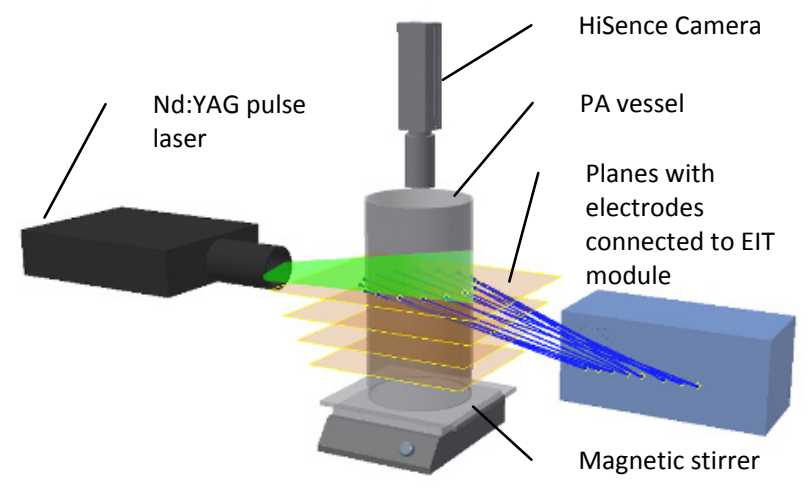

Fig. 2. Experimental setup with interrogated vessel on which there are 4 planes of electrodes for tomography and laser (left) with camera (top) for pLIF technique. 


\section{Results}

Image reconstruction technique had to be applied on measured data. The grid applied had $20 \times 20$ pixels but due to the circular dimension of the vessel only 316 pixels were used. Two reconstructions can be used: linear backprojection (LBP - this one is fast and used for real-time measurements) and sensitivity conjugate gradient (SCG) method (this one is available in a special software package for post processing).

It is necessary to emphasize that significant improvement of measured data can be achieved when a post-processing SCG method is used on the acquired data. The results presented in this paper are processed with SCG. The reconstruction generally involves two steps: a forward problem and a backward problem. In the forward problem we are inspecting changes in electrical measurements which will result when the electrical conductivity of one pixel only in the cross-section is changed by a known amount. The backward problem is then the image reconstruction process which involves surface. After approximately 5 seconds from injection we were able to see a formation of a bulk region extending from top to approximately one half of the vessel. At this point we are already able to see change in conductivity at the third plane (using tomography) where the image coming from pLIF is rather ambiguous. Further at 10 seconds from beginning clear structures in both EIT and $\mathrm{pLIF}$ images are visible. The mixture moved to the side of the vessel even no stirring was applied. Images from 3D EIT at $10 \mathrm{~s}$ and $30 \mathrm{~s}$ nicely reveal splitting of the main bulk mixture into two locations in the upper part of the vessel. Uniform mixture of the continuous substance with the injected substance is visible after $30 \mathrm{~s}$. The noncircular shape of the image for pLIF measurement is caused by the circular shape of the vessel. It arises from the refraction of the laser light at the boundaries airvessel-water. This can be avoided by using different shape of the vessel or by vessel with square cross section al outer boundary (air-vessel) and circular crosssectional inner boundary (vessel-water). Another advantage of tomography is that it is well suited for nonsquare cross-sectional vessel. The same image distortion

EIT results taken on the 3rd plane

pLIF results correspond to the 3rd EIT plane

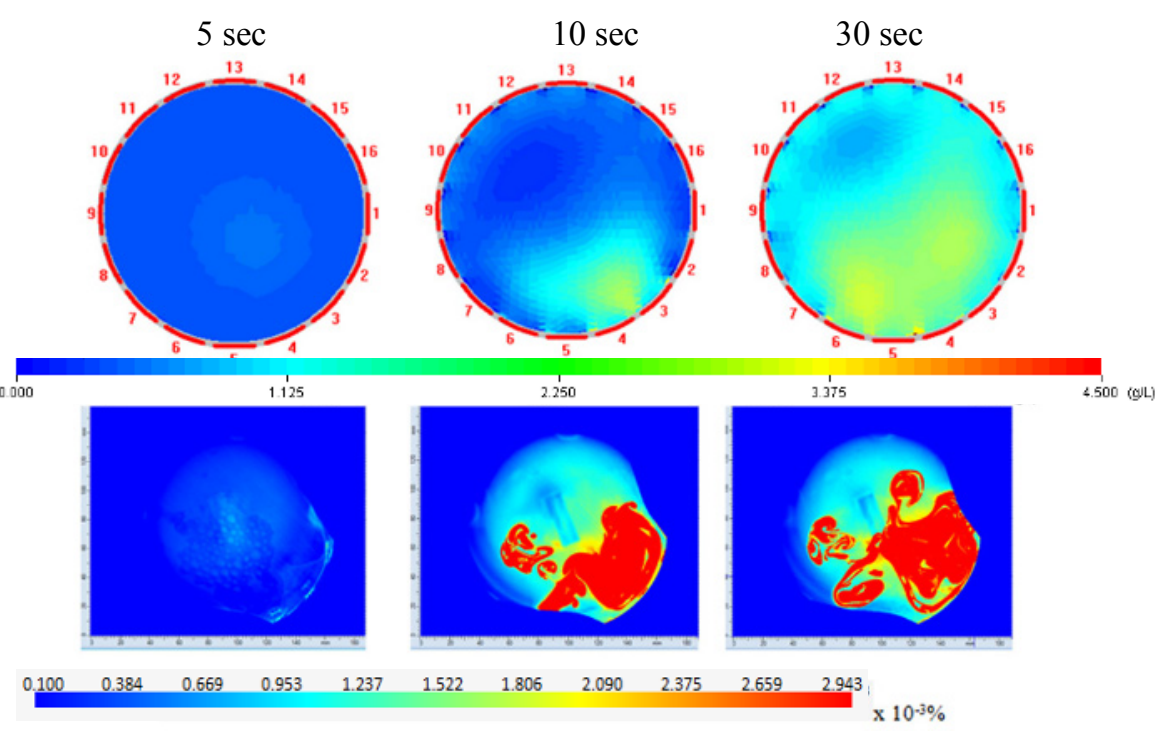

\begin{tabular}{ll}
\multicolumn{1}{c}{ 3D } & EIT results: \\
correlation & between all \\
planes &
\end{tabular}

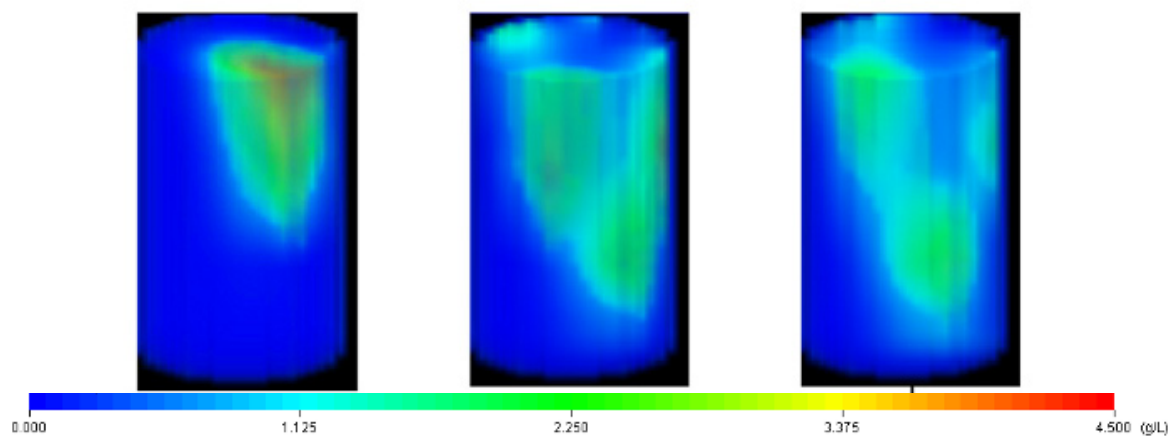

Fig. 4. Mixing process: $0.003 \% \mathrm{NaCl}$ solution in water; for $\mathrm{pLIF}$ method purpose coloured with Rhodamine $6 \mathrm{G}$ dye.

determining the electrical conductivity of each pixel within the image from the set of electrical measurements.

The measured data from the first experiment are shown in figure 4. The mixture of $\mathrm{NaCl}$ in water with Rhodame 6G was injected to the centre of the water is also visible in pLIF images in figure 4. 
In figure 5 are shown results from the second experiment. This time the magnetic stirrer was used. The substance which settled down at the bed of the vessel was more or less uniformly dispersed at each horizontal plane - see images from 10 and 20 seconds on figure 5. Due to the stirrer the substance started to be placed more at the simultaneous measurement of more planes and that the vessel and measured substance does not need to be transparent. On the other hand the drawback of is its low maxima frequency of only $50 \mathrm{~Hz}$ whereas with pLIF we can obtain much higher frequencies for fast phenomena.

EIT results taken on the 3rd plane

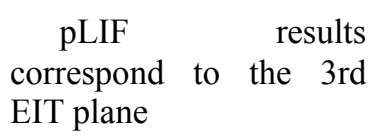

3D EIT results: correlation between all planes

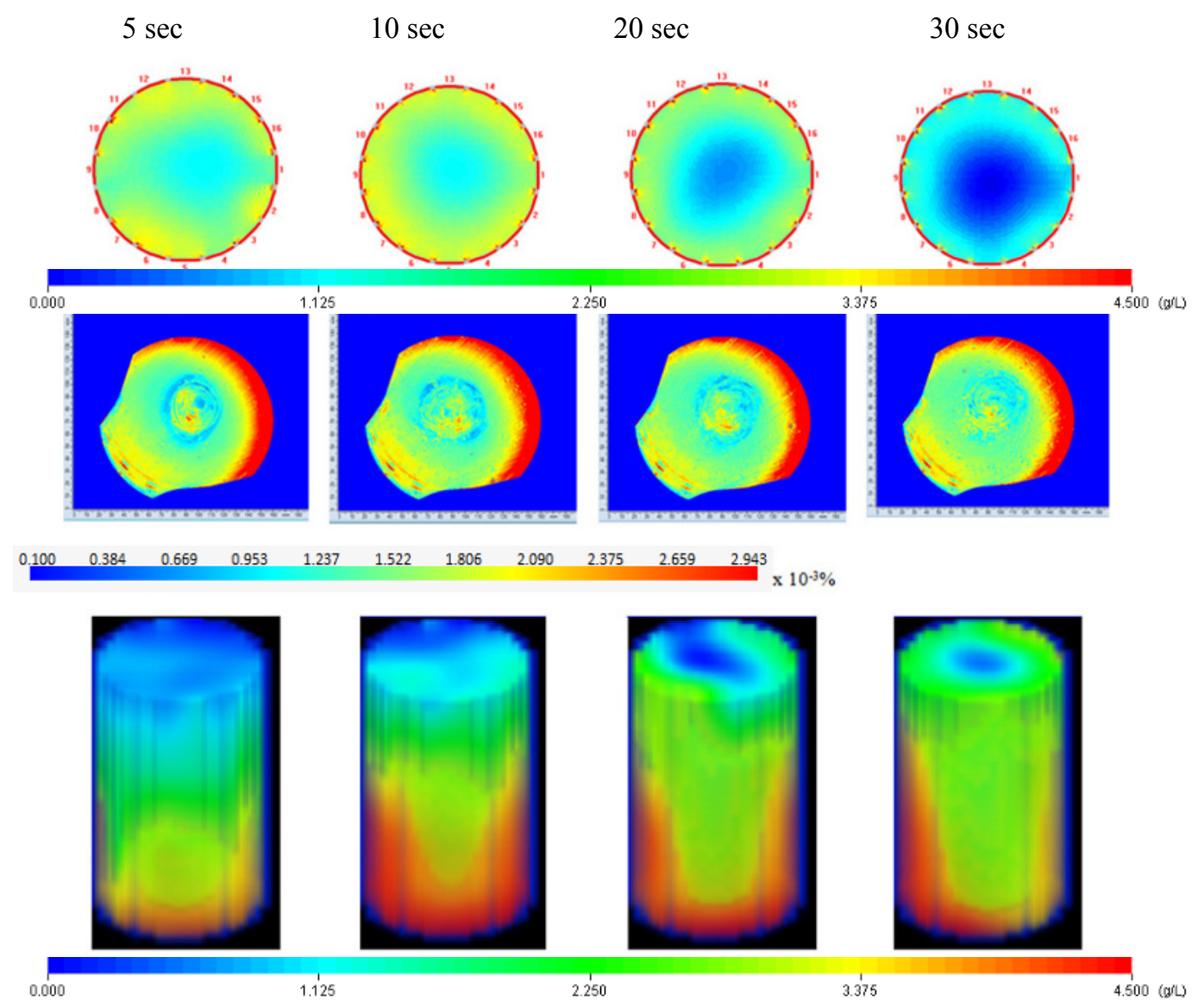

Fig. 5. Vortex structure: $\mathrm{NaCl}$ solution in water placed on magnetic stirrer $-450 \mathrm{rpm}$.

perimeter of the vessel at the same time leaving the centre of the vessel. The substance started to slightly rise to the surface of the water. At the centre of the vessel was formed a region nearly without the substance - see at 40 seconds. From the pLIF images it is nicely visible the rotational movement of the water in the vessel whereas from the EIT measurements (especially from 3D images) is more visible a non-uniform vortex formation $(10 \mathrm{~s}, 30$ s) which at the end (40 s) transforms into axial symmetric. This behaviour makes senesce since it takes some time to observe full water stirring due to a stirrer.

\section{Conclusions}

We have shown that results coming from pLIF and EIT are comparable. Further investigation has to be undertaken in the area of analysis of tomographic images. Since p2+ enables exporting into *.vtk file format - nice way how to process data could be a software MayaVi. Big advantage of tomography over pLIF method lies in

\section{Acknowledgements}

Authors gratefully thank to the support of Technical university of Liberec - the student grant support and the research projects CZ.1.05/2.1.00/01.0005 a CZ.1.07/2.3.00/30.0024.

\section{References}

1. D. Chen, C. Yang, G. Zheng, X. Yu, L. Sun, Front. Mech. Eng. China, 2 (2006)

2. M. Goharian, M. Soleimani, A. Jegatheesan, K. Chin, G. R. Moran, Annals of Biomedical Engineering, 36 (2008)

3. K. Cho, S. Kim, KSME International Journal, 13 (1999)

4. K. Primrose, C. Qiu, 1st World Congress on Industrial Process Tomography, (1999) 